\title{
Note on some revelations in prioritization, theory of choice and decision making support methodology
}

\begin{abstract}
Pawel Tadeusz Kazibudzki
The Faculty of Social Sciences, Jan Dlugosz University, Waszyngtona 4/8, 42-200 Czestochowa, Poland. E-mail: emailpoczta@gmail.com. Tel: +48.60.46.31.997.

Accepted 8 October, 2012

Providing unique answers for any multicriteria decision making support methodology seems to be a crucial issue not only from the perspective of its creditability but also the entire concept validity. However, it is known that the analytic hierarchy process (AHP), as the prioritization and choice theory, can provide different priority vectors, depending on the method applied during the realization of the process. It is also the fact that we are being convinced from the very long time that only one approach in this matter is the only one and no other qualifies (principal right eigenvector method). However, this approach finds itself to have serious drawbacks and some flaws. This paper reveals some fundamental discoveries within this field thanks to seminal computer simulations for the entire AHP framework (as opposed to single matrix simulation research). The result concurs with the opinion that the right eigenvector method is not unique as long as reciprocal pairwise comparison matrices are concerned (what is embedded in the AHP process) and when it could be (nonreciprocal cases) it is not able to due to lack of inconsistency measure, which is an indispensable element of the entire AHP concept.
\end{abstract}

Key words: Decisions making support methodology, analytic hierarchy process, principal right eigenvector, priority vector, constrained optimization.

\section{INTRODUCTION}

\section{Judgment and decision making}

Managerial live is the sum of their decisions. Frequently, the moment when they decide is equally important to the fact what they decide. Everyday business life and its history are full of lessons that can help to recognize that crucial event. It seems that decision making is the central activity of managerial brain which determines meaning and set priorities. It occurs that there is little doubt at all that the human brain performs prioritization (Saaty, 2000). Our central nervous system is continuously faced with the task of making decisions on the basis of information about the outside world. We see the world bottom up, from the more detailed and complex to the more abstract and integrated. Synthesis then is a fundamental process of decision making, in which the priorities of many different elements are integrated into one meaningful outcome that represents or highlights the underling order which is supposed to be evaluated. It has been noticed that many different levels of synthesis can be integrated only if the representations are made on relative ratio scales. It is claimed that most powerful everyday manifestation of human being capability is judgment, which is done by making pairwise comparisons. By comparing, human being can determines which of the two elements has more of a property that the elements have in common, and how much more. Judgments for each pair of elements are then added to extract relative priorities on the ratio scale for the many elements that are compared in pairs. The analytic hierarchy process (AHP) is a decision making support methodology based on these very same principles, and that makes it a very powerful and a "user friendly" concept. It derives ratio scales from paired comparisons. It possesses one procedure that preserves ranks absolutely as required by the invariance principle (ideal mode), and the other which allows ranks to change (distributive mode). In the absolute measurement mode of the AHP, one derives priorities for different intensities of each criterion. One then divides by the priority of the largest intensity in each case and then creates an ideal alternative, the best imaginable choice. In this case each 
alternative takes its place in the ranking and has no effect on the rank of the other alternatives. Rank is then always preserved. But the AHP is also concerned with those situations where ranks can and should change. The distributive mode of the AHP produces preference scores by normalizing the performance scores. In other words, in this mode one must follow different procedure for ranking which takes the performance score received by each alternative and divides it by the sum of performance scores of all alternatives under given criterion. Thus, priorities are derived for the criteria in terms of their importance to achieve the goal, and for the performance of the alternatives on each criterion. These priorities are derived based on pairwise assessment using judgment. Finally, a weighting and adding process is used to obtain overall priorities for the alternatives as to how they contribute to the goal. Thus, one can say the AHP can be considered to be both a descriptive and prescriptive model of decision making. It is also the most widely used decision making approach in the world today, as well most validated methodology (thousands of actual applications in which the AHP results were accepted and used by the competent decision makers). Among the applications, the most recently reported in literature may list supplier selection (Chamodrakas et al., 2010; Kilincci and Onal, 2011), energy selection (Kahraman and Kaya, 2010), strategy selection (Chen and Wang, 2010; Li and $\mathrm{Li}, 2009$ ), comparison of bridge designs (Farkas, 2011), developing passenger train schedules (Isaai et al., 2011), ranking university majors (Rad et al., 2011) or project complexity evaluation (Vidal et al., 2011) - to name just a few articles from very recent years. More thorough reviews of the contemporary applications and developments in AHP can be found in researches done by Ishizaka and Labib (2011), Ho (2008) and Vaidya and Kumar (2006). However, a great number of books have been written by different authors on the analytic hierarchy process (Bhushan and Ria, 2004; Hummel, 2001; Saaty and Vargas, 2000, 2006; Schmoldt et al., 2001; Saaty, 2006, 2001, 2000).

\section{Notations and principles of the analytic hierarchy process}

The AHP is grounded on the well-defined mathematical structure of consistent matrices and their associated right-eigenvector's ability to generate true or approximate weights (Merkin, 1979; Saaty, 1990). Generally speaking a problem of deriving priority weights from so called pairwise comparison matrix (PCM) denoted as $A(a)=\left[a_{i j}\right]_{\times \times n}$ with elements $a_{i j}=a_{i} / a_{i}$ is to estimate a priority vector (PV) $w=\left[w_{1}, w_{2}, w_{3}, \ldots, w_{n}\right]^{\top}$ on the bases of the matrix $A(a)$ which comprises a decision maker (DM) pairwise comparisons judgments concerning the importance of a given binary set of alternatives. Commonly the priority weights $w_{i}, i=1, \ldots, n$, are chosen to be positive and normalized to unity: $\sum_{i}^{n} w_{i}=1$, and the elements $a_{i j}$ of the matrix $A(a)$ are then the DM judgments about the priority ratios $w_{i} / w_{j}, i, j=1, \ldots, n$, where $n$ is the number of all alternatives being considered. In a perfect judgment case then, we have:

$A \times w=n \times w$

and in this situation the PV $w$ can be computed by solving that so called eigenvector equation. It is so because the number $n$ in the perfect case (matrix $A(a)$ is consistent) is the principal eigenvalue of $A(a)$, that is the largest solution of the characteristic equation:

$\operatorname{det}(A-l a m b d a \times I)=0$

where I denotes identity matrix of order $n$. In this case it is also the only nonzero eigenvalue. On the other hand, when the case is not perfect (matrix $A(a)$ is inconsistent) an estimate of the true PV is normalized right eigenvector (REV) associated with the largest eigenvalue. Thus, in order to obtain the estimate we need to solve general eigenvector equation:

$A \times w=l a m b d a_{\max } \times w$

where lambda $a_{\max }$ denotes the principal eigenvalue which is not smaller than $n$, is simple and its existence is guaranteed by Perron-Frobenius theorem, while other lambdas are close to zero. If the elements of a matrix $A(a)$ satisfy the condition $a_{i j}=1 / a_{j i}$ for all $i, j=1, \ldots, n$ then the matrix $A(a)$ is said to be reciprocal (RPCM). If its elements satisfy the condition $a_{i k} a_{k j}=a_{i j}$ for all $i, j, k=1, \ldots, n$ and the matrix is reciprocal, then it is called consistent. Finally, the matrix $A(a)$ is said to be transitive (TPCM), if the following conditions hold: (i) if for any $I=1, \ldots, n$, an element $a_{l j}$ is not less than an element $a_{l k}$ then $a_{i j} \geq a_{i k}$ for $i=1, \ldots, n$, and (ii) if for any $l=1, \ldots, n$, an element $a_{j l}$ is not less than an element $a_{k l}$ then $a_{j i} \geq a_{k i}$ for $i=1, \ldots, n$. Obviously, in the case of the reciprocal PCM the two conditions (i) and (ii) are equivalent.

Fundamentally, all theories are based on axioms, so is the AHP. It has to conform to three relatively simple axioms: reciprocal axiom, homogeneity axiom and independence axiom (Saaty, 1986). Reciprocity simply means that for example if an element $a$ is 3 times larger than an element $b$, then an element $b$ is one third as large as an element $a$, whereas homogeneity axiom states that the elements being compared should not differ by too much, otherwise there are larger errors in judgments. So, when constructing a hierarchy of objectives, a decision maker should make the effort to arrange elements in clusters in that way they did not differ by more than an order of magnitude in any cluster. Finally, the last axiom states that judgments about, or the 
priorities of, the elements in a hierarchy do not depend on lower level elements. Most AHP theorists and practitioners believe that AHP's axioms are simpler and more realistic than those concerning other decision theories including the most competitive to AHP - multi attribute utility theory (MAUT). However, this polemics is beyond the scope of this research. Basically, there are two kinds of transitivity: ordinal and cardinal. The first one is that if an alternative $a$ is preferred to an alternative $b$ and $b$ is preferred to $c$, then an alternative a must be preferred to an alternative $c$. The second is that if an alternative $a$ is preferred to an alternative $b$ two times and $b$ is preferred to $c$ four times, then an alternative a must be preferred to an alternative $c$ eight times. The latter requirement is the stronger one, and it implies consistency. This also means that consistent matrix is cardinally transitive what entails its ordinal transitivity. An inconsistent matrix may be neither cardinally nor ordinally transitive and can be expressed in the following form:

$a_{i j}=e_{i j} \times w_{i j}$

where $e_{i j}$ is a perturbation factor which is expected to be near 1 (Saaty, 2003; Sun and Greenberg, 2006; Ishizaka and Labib, 2011). In a statistical approach and many simulation studies the perturbation factor is interpreted as a realization of a random variable (Zahedi, 1986). It is important to notice that the methodology of capturing the degree of PCM inconsistency in order to derive a vector of priorities is a central point of the AHP and a crucial issue for the whole priority theory. Obviously, significant violations of the consistency may be misleading about the value of information obtained making the methodology simply useless. As far it is concerned, the most popular measure of PCM inconsistency belongs to Saaty (1980) and is strictly related to REV, what makes it especially attractive. The point here is that there is quite few propositions in the literature for consistency measures, e.g. Grzybowski's inconsistency index (Grzybowski, 2010), Koczkodaj's inconsistency index (Bozóki and Rapcsák, 2008), a residual mean square connected with logarithmic least squares method (LLSM), known also as geometric mean procedure (GM) (Budescu et al., 1986; Crawford and Williams, 1985; Aguaron and MorenoJimenez, 2003), and others (Bryson, 1995; Saaty and Vargas, 1984). However, it is argued that so long as inconsistency is tolerated, REV is the basic theoretical concept for deriving a scale and no other methods qualify, what entails the concern about the usefulness of inconsistency measures derived from them. Although, during the last three decades plenty of other methods have been proposed: beginning with the most popular one, already mentioned the geometric mean procedure (Crawford and Williams, 1985; Cook and Kress, 1988; Saaty and $\mathrm{Hu}, 1998)$, and other methods based on constrained optimization models (Hovanov et al., 2008; Lam and Choo, 1995), including least squares method
(Lin, 2007; Zahedi, 1986), and various versions of goal programming methods (Bryson, 1995; Grzybowski, 2010; Lin, 2006; Moy et al., 1997), through methods based on some statistical concepts (Basak, 1998; Dong et al., 2008; Lipovetsky and Tishler, 1997), ending on methods based on fuzzy preference description (Xu, 2004).

\section{Definition of the problem}

It is indispensable for a reliable decision making support methodology to provide unique answers for the alternatives of a decision. As it was presented above a variety of methods and algorithms were created in order to derive true priority vectors from intuitive judgments. When judgments are more or less consistent, the results of all approaches rather coincide. However, in real life, judgments are permanently inconsistent. Such a situation gives rise to different priority vectors due to application of different methods. It was also demonstrated that especially in multicriteria processes even when different methods provide priority vectors that are close to each other, both on criteria and alternatives, after synthesis according to a well-prescribed procedure (Saaty, 1980) (standard AHP aggregation based on weighting and adding) the rank order of the alternatives can vary (Saaty and $\mathrm{Hu}, 1998)$. One could come up with the idea that such abundance of potential results that a prospective decision maker can obtain violates the uniqueness requirement mentioned earlier and therefore seems unacceptable. On the other hand, it is disseminated that principal right eigenvector method (REV) captures transitivity in matrices that are not consistent in the authentic way. That could lead to a conclusion that maybe the REV is necessary and sufficient to facilitate credible decision making based on priority weighting followed by inconsistent matrices comprising pairwise comparison judgments. However, let it be remembered that REV, despite of obvious advantages, has also few drawbacks and flaws. First of all, Saaty's procedure does not optimize any performance criterion, so it cannot be interpreted in statistical or optimization fashion, thus it is difficult to compare resulting PVs with the ones elicited with the help of other methods (Bryson, 1995; Budescu et al., 1986; Crawford and Williams, 1985). Furthermore, unlike many optimization techniques, the REV does not allow the DM to introduce any additional constraints for the priority vector which, according to decision-maker beliefs, should be satisfied by the weights (Bana e Costa and Vansnick, 2008; Belton and Gear, 1983; Lipovetsky and Conklin, 2002). Moreover, the REV should be used only for reciprocal PCMs, at least in the case when one needs to use Saaty's consistency index, which is meaningful exclusively with reciprocal PCMs, otherwise inexplicable. As a result, in practice reciprocity of PCMs is a very popular requirement, although many authors argue that it is an artificial condition which impoverishes 
the PCM about information concerning the unknown priority vector that otherwise could have been revealed (Basak, 1998; Budescu et al., 1986; Hovanov et al., 2008; Lipovetsky and Tishler, 1997; Zahedi, 1986).

\section{RESEARCH GENESIS AND ITS METHODOLOGY}

\section{Methodical framework}

Although the problem remains blurred, the only way to clarify it, in our opinion, is to proceed with simulations not commonly known in literature. It should be concerned with only one single PCM and comprise the entire program within a given AHP framework, which consists of different number of criteria and alternatives. Thus, the objective of this section is to examine the performances of the REV and two other arbitrarily chosen prioritization procedures within a given AHP framework. Thus, in order to serve this purpose the following procedures were chosen:

- (SRDM) that is sum of squared relative differences (Grzybowski, 2012), given by the formula:

$\min \operatorname{SRDM}(w)=\sum_{i=1}^{n}\left(\frac{1}{n w_{i}} \sum_{j=1}^{n} a_{i j} w_{j}-1\right)^{2}$

Subject to: $\sum_{j=1}^{n} w_{j}=1, \quad w_{i}>0, \quad i, j=1, \ldots, n$

- (REV) that is principal right eigenvector method (Saaty, 1980), described earlier in this paper,

- (LUA) that is logarithmic utility approach being author's refinement of SRDM concept and given by the formula:

$\min \mathrm{LUA}=\sum_{i=1}^{n}\left(\operatorname{In}\left(\sum_{j=1}^{n} \frac{a_{i j} \times w_{j}}{n \times w_{i}}\right)\right)^{2}$

subject to: $\sum_{j=1}^{n} w_{j}=1, \quad w_{i}>0, \quad i, j=1, \ldots, n$

The comparison of indicated procedures performances for the entire AHP framework is going to be based on the following novel assumptions. We assume the hierarchy consists of three levels: goal, criteria and alternatives as in Saaty and Hu (1998), which is supposed to reflect the hypothetic case of real managerial decision problem. Then, in order to compare the accuracy of the estimations obtained by chosen procedures we simulate different situations related to various sources of the PCM inconsistency. Fundamentally, the inconsistency commonly results from errors caused by the nature of human judgments and errors arisen from the technical realization of the comparison procedure. The latter ones, appear mainly due to errors resulting from the forced reciprocity requirement and so called rounding errors which are related to the numerical ratio scale whose values should be used by prospective decision makers in order to express somehow their judgments (Dong et al., 2008; Lipovetsky and Tishler, 1997; Lipovetsky and Conklin, 2002). Obviously, in conventional AHP applications the most popular is Saaty's numerical scale which comprises the integers from 1 to 9 and their reciprocals. And this exhaust the problem of errors caused by the technical realization of the comparison procedure.

But there are also errors caused by the nature of human judgments which we should also take into account. Fortunately, they can be represented as the realization of some random process in accordance with the formula [4]. Probability distributions of the perturbation factor $e_{i j}$ mainly involve uniform and gamma, as well truncated normal or log-normal (Basak, 1998; Choo and Wedley, 2004; Lin, 2007; Zahedi, 1986). In order to clarify the novelty of the simulation framework we are going to describe now the procedure scenario.

In our simulations, we generate uniformly random and normalized 'ideal' priority vector for uniformly randomly chosen number of criteria. Next, we generate uniformly random and normalized 'ideal' priority vectors of alternatives for the given set of criteria with uniformly randomly chosen number of alternatives. Then, we calculate the 'ideal' total priority vector (ITPV) of weights, according to a well-prescribed procedure (standard AHP aggregation based on weighting and adding). Next, on the bases of 'ideal' individual priority vectors generated for the given set of criteria and the given sets of alternatives, we create correspondent pairwise comparison matrices (PCMs). Then, we make them inconsistent in three ways, either only through rounding their elements to Saaty's scale or exclusively through perturbation of their elements in accordance with relation [4] or doing both these things successively perturbation and then rounding. Next, on the bases of such created inconsistent PCMs we compute their respective priority vectors with the application of chosen methods: SRDM, REV and LUA. Then, for each method we calculate the total priority vector (TPV) applying standard AHP aggregation procedure. Finally, we compare values of such obtained TPV with the values of original ITPV. This scenario is iterated assumed number of times, in our simulation study we repeated the scenario ten thousands times.

In order to evaluate the performance of chosen methods, we compute known from literature: the Pearson correlation coefficient (PCC) between the 'ideal' priority vector and its estimates, Spearman rank correlation coefficient (SRCC) (Moy et al., 1997; Budescu et al., 1986), and mean absolute deviation (Choo and Wedley, 2004; Lin, 2007; Dong et al., 2008) which is defined by the following formula:

$$
\operatorname{MAD}(w, \hat{w})=\frac{1}{n} \sum_{i=1}^{n}\left|w_{i}-\hat{w}_{i}\right|
$$

where $w$ and $\hat{W}$ denote the 'ideal' priority vector and its estimate, consecutively.

In our simulations, we consider two approximation options: with and without forced reciprocity. When forced reciprocity condition is applied, the perturbed PCM inputs are taken only from above its diagonal elements, and the remaining ones are entered as the inverses of the corresponding symmetric units in relation to its diagonal elements. We denote matrices with forced reciprocity condition applied as FRPCMs, whereas the remaining ones as APCMs (arbitrary pairwise comparisons matrices).

\section{Methodical example within simplified framework}

Our example describes the simplified simulation procedure in the situation when only one PCM is considered as opposite to the whole AHP framework. The idea of this example is to clarify the methodology lying behind the description introduced in former subchapter of this paper. So, we begin with the assumption that we know the 'ideal' priority vector. Let the 'ideal' normalized priority vector be imposed as follows: $w=[0.5,0.35,0.15]^{\top}$. Then, the 
'ideal' matrix $A(w)$ derived on the bases of this vector has the following entries:

$$
A(w)=\left[\begin{array}{ccc}
1 & 1.42857 & 3.3333 \\
0.7 & 1 & 2.3333 \\
0.3 & 0.428571 & 1
\end{array}\right]
$$

The idea is now to perturb such obtained consistent matrix to obtain an inconsistent one. We can do it in three ways: perturb its entries following the relation [4], round its entries to Saaty's numerical scale or both perturb its entries following the relation [4] and then round its entries to Saaty's numerical scale. Here, for the purpose of simplification, we will proceed only with steps constrained to direct rounding (without earlier perturbation in accordance to relation [4]) as the representation of technical realization of AHP methodology. We have then:

$$
A(a)=\left[\begin{array}{ccc}
1 & 1 & 3 \\
1 / 2 & 1 & 2 \\
1 / 3 & 1 / 2 & 1
\end{array}\right]
$$

Due to rounding errors, we receive nonreciprocal PCM, which can be left as it is or can be made as a reciprocal. Following the procedure described earlier in this paper the forced reciprocal PCM (FRPCM) we would want to obtain could have the following entries:

$$
\tilde{A}(a)=\left[\begin{array}{ccc}
1 & 1 & 3 \\
1 & 1 & 2 \\
1 / 3 & 1 / 2 & 1
\end{array}\right]
$$

Now, on the bases of this matrix, which can be perceived as a decision maker best attempt to approximate the 'ideal' $A(w)$ we can calculate the priority vectors with the application of SRDM, REV and LUA procedures, respectively. Then, we can compare such obtained results with the primary 'ideal' $\mathrm{PV}$ and compute basic statistics such as MAD, SRCC, and PCC. The results of such calculations and priority vectors received with the application of chosen proposed methods in the case of FRPCM and APCM are presented in Tables 1 and 2.

As we can notice in the presented example all methods deliver the same ranks for priorities what is reflected by SRCC $=1$ for all of them regardless of the fact the forced reciprocity was applied or not. In order to check if this is true for the entire AHP framework with different number of criteria and alternatives we can simulate similar scenarios with the application of different probability distributions for perturbation factor in the relation [4] and different size of errors reflected by the range of perturbation factor in the equation [4].

\section{PRELIMINARY SIMULATION RESULTS}

Following the example (Saaty and $\mathrm{Hu}$, 1998) illustrating the case where variability in ranks does not occur for each individual judgment matrix, but still occurs in the overall ranking of the final alternatives due to the multicriteria process itself we proceed with respectively designed simulations (assuming the same AHP framework as in the cited example, that is goal, four criteria and four alternatives). We simulated 100 such AHP models, making them inconsistent 100 times in each case. The inconsistency was either exclusively due to rounding errors or exclusively due to perturbation factor $\left(e_{i j}\right)$ drawn uniformly from the interval $e_{i j} \in[0.5,1.5]$, or both (perturbbation and rounding). Thus, Tables 3,4 and 5 present average performances of chosen methods for ten thousands cases (one hundred uniformly random AHP frameworks, each perturbed one hundred times with the application of certain procedure).

\section{DISCUSSION WITH PRIMARY SIMULATION OUTCOMES}

From the simulation results obtained concerning inconsistent cases, judging on the bases of SRCC, all methods are very competitive. However, in the situations when reciprocity is forced the REV no longer prevails, but minimally concedes one or both chosen procedures. Let us underline, that the REV prevails only in the situation when reciprocity of PCM is not required but in this situation recedes in other field because its inconsistency index cannot longer operate in these circumstances as it provides no information about judgments consistency (a crucial issue in the AHP).

Then, taking into account the entire simulation scenario it becomes obvious that it is not justified to claim that the REV is the dominant method among others as it was done by Saaty and Hu (1998). Additionally, exceptionally important seems to be the fact that the REV is as the rule worse in its performance, measured by SRCC as long as reciprocity of PCMs is forced which is the embedded norm in the AHP. It means that the REV is not as effective as other arbitrarily chosen methods presented here in the process of unique capturing the ratio scale rank order inherent in inconsistent pairwise comparison judgments as long as reciprocity is forced what stands in opposition to the theorem presented by Saaty and $\mathrm{Hu}$ (1998).

To elaborate on this further we examine now the average performances results for ten thousands cases (100 AHP frameworks, each perturbed 100 times) but this time only for reciprocal PCMs (FRPCMs). The inconsistency will be imposed this time due to rounding errors combined with perturbation factor $\left(e_{i j}\right)$ drawn uniformly, log-normally, truncated normally or gamma from imposed different intervals. Additionally, this time the number of alternatives and criteria for each model will also be drawn uniformly from different intervals. The simulations results are presented in the consecutive tables (Tables 6 to 13).

Noticeably, taking into account reciprocal PCMs, and basing the judgment on SRCC as the indicator of 'ideal' priority vector rank preservation and possibility to recover it as accurately as possible due to certain procedure application. It can be notice that the REV is always 
Table 1. Comparison of SRDM, REV and LUA performances in the example with rounding errors and forced reciprocity applied for 'ideal' $P V: ~ w=[0.5,0.35,0.15]^{\top}$.

\begin{tabular}{llccc}
\hline \multirow{2}{*}{ Method } & \multirow{2}{*}{ Estimate } & \multicolumn{3}{c}{ Performance measure } \\
\cline { 3 - 5 } & & MAD & SRCC & PCC \\
\hline SRDM & {$[0.443429,0.387371,0.1692]^{\top}$} & 0.0377139 & 1 & 0.969744 \\
LUA & {$[0.443429,0.387371,0.1692]^{\top}$} & 0.0377139 & 1 & 0.969744 \\
REV & {$[0.443429,0.387371,0.1692]^{\top}$} & 0.0377139 & 1 & 0.969744 \\
\hline
\end{tabular}

Table 2. Comparison of SRDM, REV and LUA performances in the example with rounding errors and without forced reciprocity applied for 'ideal' PV: $w=[0.5,0.35,0.15]^{\top}$.

\begin{tabular}{llccc}
\hline \multirow{2}{*}{ Method } & \multirow{2}{*}{ Estimate } & \multicolumn{3}{c}{ Performance measure } \\
\cline { 3 - 5 } & & MAD & SRCC & PCC \\
\hline SRDM & {$[0.483379,0.333015,0.183606]^{\top}$} & 0.022404 & 1 & 0.996463 \\
LUA & {$[0.483518,0.333119,0.183362]^{\top}$} & 0.0222415 & 1 & 0.996514 \\
REV & {$[0.484669,0.333977,0.181354]^{\top}$} & 0.0209025 & 1 & 0.996911 \\
\hline
\end{tabular}

Table 3. Performance evaluations of chosen three different methods for 10,000 cases within AHP framework $\left(n_{k}=n_{a}=4\right)$ exclusively with the application of rounding errors.

\begin{tabular}{lcccccc}
\hline \multirow{2}{*}{ Method } & \multicolumn{3}{c}{ FRPCM } & \multicolumn{3}{c}{ APCM } \\
\cline { 2 - 7 } & MAD & SRCC & PCC & MAD & SRCC & PCC \\
\hline SRDM & 0.0152269 & 0.963095 & 0.996955 & 0.0137668 & 0.967400 & 0.997459 \\
REV & 0.0151595 & 0.963075 & 0.996973 & 0.0136607 & 0.967835 & 0.997509 \\
LUA & 0.0152334 & 0.963115 & 0.996953 & 0.0137689 & 0.967340 & 0.997460 \\
\hline
\end{tabular}

Table 4. Performance evaluations of chosen three different methods for 10,000 cases within AHP framework $\left(n_{k}=n_{a}=4\right)$ exclusively with the application of perturbation factor drawn uniformly from the interval $e_{i j} \in[0.5,1.5]$.

\begin{tabular}{lcccccc}
\hline \multirow{2}{*}{ Method } & \multicolumn{3}{c}{ FRPCM } & \multicolumn{3}{c}{ APCM } \\
\cline { 2 - 7 } & MAD & SRCC & PCC & MAD & SRCC & PCC \\
\hline SRDM & 0.0177456 & 0.94710 & 0.993994 & 0.0133314 & 0.95754 & 0.996406 \\
REV & 0.0162058 & 0.94708 & 0.994137 & 0.0127045 & 0.95832 & 0.996595 \\
LUA & 0.0162051 & 0.94718 & 0.994140 & 0.0128606 & 0.95778 & 0.996504 \\
\hline
\end{tabular}

Table 5. Performance evaluations of three different methods for 10,000 cases within AHP framework $\left(n_{k}=n_{a}=4\right)$ with the application of both perturbation factor drawn uniformly from the interval $e_{i j} \in[0.5,1.5]$ and rounding errors.

\begin{tabular}{lcccccc}
\hline \multirow{2}{*}{ Method } & \multicolumn{3}{c}{ FRPCM } & \multicolumn{3}{c}{ APCM } \\
\cline { 2 - 7 } & MAD & SRCC & PCC & MAD & SRCC & PCC \\
\hline SRDM & 0.0247155 & 0.93454 & 0.990383 & 0.0195971 & 0.94708 & 0.994080 \\
REV & 0.0246628 & 0.93454 & 0.990397 & 0.0193604 & 0.94798 & 0.994319 \\
LUA & 0.0247214 & 0.93456 & 0.990382 & 0.0195377 & 0.94724 & 0.994144 \\
\hline
\end{tabular}

dominated by one of two methods presented here.

In order to present mean performance results of SRDM, LUA and REV for the entire AHP framework we adopted and upgraded simulation frameworks already proposed in the literature by Basak (1998), Choo and Wedley (2004), Lin (2007), Grzybowski (2012) and 
Table 6. Performance evaluations of chosen three different methods for 10,000 cases within AHP uniformly random framework $\left(n_{k}, n_{a} \in[5,9]\right)$ with the application of both perturbation factor drawn uniformly from the interval $e_{i j} \in[0.1,1.9]$ and rounding errors.

\begin{tabular}{lccc}
\hline & \multicolumn{3}{c}{ FRPCM } \\
\cline { 2 - 4 } Method & MAD & SRCC & PCC \\
\hline SRDM & 0.0287422 & 0.821174 & 0.943263 \\
REV & 0.0288576 & 0.818528 & 0.942239 \\
LUA & 0.0287142 & 0.821731 & 0.943471 \\
\hline
\end{tabular}

Table 7. Performance evaluations of chosen three different methods for 10,000 cases within AHP uniformly random framework $\left(n_{k}, n_{a} \in[3,7]\right)$ with the application of both perturbation factor drawn uniformly from the interval $e_{i j} \in[0.05,1.95]$ and rounding errors.

\begin{tabular}{lccc}
\hline \multirow{2}{*}{ Method } & \multicolumn{3}{c}{ FRPCM } \\
\cline { 2 - 4 } & MAD & SRCC & PCC \\
\hline SRDM & 0.0421448 & 0.821411 & 0.920532 \\
REV & 0.0423495 & 0.818026 & 0.919355 \\
LUA & 0.0420947 & 0.822583 & 0.920789 \\
\hline
\end{tabular}

Table 8. Performance evaluations of chosen three different methods for 10,000 cases within AHP uniformly random framework $\left(n_{k}, n_{a} \in[8,12]\right)$ with the application of both perturbation factor drawn log-normally from the interval $e_{i j} \in[0.05,1.95]$ and rounding errors.

\begin{tabular}{lccc}
\hline \multirow{2}{*}{ Method } & \multicolumn{3}{c}{ FRPCM } \\
\cline { 2 - 4 } & MAD & SRCC & PCC \\
\hline SRDM & 0.0240699 & 0.738000 & 0.885866 \\
REV & 0.0242049 & 0.736278 & 0.884172 \\
LUA & 0.0240361 & 0.738273 & 0.886230 \\
\hline
\end{tabular}

Table 9. Performance evaluations of chosen three different methods for 10,000 cases within AHP uniformly random framework $\left(n_{k}, n_{a} \in[3,7]\right)$ with the application of both perturbation factor drawn log-normally from the interval $e_{i j} \in[0.05,1.95]$ and rounding errors.

\begin{tabular}{lccc}
\hline \multirow{2}{*}{ Method } & \multicolumn{3}{c}{ FRPCM } \\
\cline { 2 - 4 } & MAD & SRCC & PCC \\
\hline SRDM & 0.0536403 & 0.811334 & 0.896854 \\
REV & 0.0538714 & 0.809600 & 0.895606 \\
LUA & 0.0535864 & 0.811654 & 0.897116 \\
\hline
\end{tabular}

Zahedi (1986). For instance in the simulation framework described by Choo and Wedley (2004), it was proposed to study two types of PCMs: the PCMs that contain many small errors and the PCMs that contain one large error. In the revised framework described by Lin (2007), proposed
Table 10. Performance evaluations of chosen three different methods for 10,000 cases within AHP uniformly random framework $\left(n_{k}, n_{a} \in[8,12]\right)$ with the application of both perturbation factor drawn truncated normally from the interval $e_{i j} \in[0.75,1.25]$ and rounding errors.

\begin{tabular}{lccc}
\hline \multirow{2}{*}{ Method } & \multicolumn{3}{c}{ FRPCM } \\
\cline { 2 - 4 } & MAD & SRCC & PCC \\
\hline SRDM & 0.00656960 & 0.974134 & 0.997312 \\
REV & 0.00647995 & 0.974105 & 0.997360 \\
LUA & 0.00657727 & 0.974142 & 0.997308 \\
\hline
\end{tabular}

Table 11. Performance evaluations of chosen three different methods for 10,000 cases within AHP uniformly random framework $\left(n_{k}, n_{a} \in[3,7]\right)$ with the application of both perturbation factor drawn truncated normally from the interval $e_{i j} \in[0.05,1.95]$ and rounding errors.

\begin{tabular}{lccc}
\hline \multirow{2}{*}{ Method } & \multicolumn{3}{c}{ FRPCM } \\
\cline { 2 - 4 } & MAD & SRCC & PCC \\
\hline SRDM & 0.0242055 & 0.918557 & 0.988582 \\
REV & 0.0241595 & 0.918383 & 0.988553 \\
LUA & 0.0242102 & 0.918700 & 0.988587 \\
\hline
\end{tabular}

Table 12. Performance evaluations of chosen three different methods for 10,000 cases within AHP uniformly random framework $\left(n_{k}, n_{a} \in[3,7]\right)$ with the application of both perturbation factor drawn gamma from the interval $e_{i j} \in[0.75,1.25]$ and rounding errors.

\begin{tabular}{lccc}
\hline \multirow{2}{*}{ Method } & \multicolumn{3}{c}{ FRPCM } \\
\cline { 2 - 4 } & MAD & SRCC & PCC \\
\hline SRDM & 0.01838324 & 0.948931 & 0.991685 \\
REV & 0.0183054 & 0.948900 & 0.991729 \\
LUA & 0.0183912 & 0.948911 & 0.991679 \\
\hline
\end{tabular}

Table 13. Performance evaluations of chosen three different methods for 10,000 cases within AHP uniformly random framework $\left(n_{k}, n_{a} \in[8,12]\right)$ with the application of both perturbation factor drawn gamma from the interval $e_{i j} \in[0.05,1.95]$ and rounding errors.

\begin{tabular}{lccc}
\hline \multirow{2}{*}{ Method } & \multicolumn{3}{c}{ FRPCM } \\
\cline { 2 - 4 } & MAD & SRCC & PCC \\
\hline SRDM & 0.0321617 & 0.632952 & 0.741426 \\
REV & 0.0322768 & 0.628579 & 0.741102 \\
LUA & 0.0321762 & 0.633529 & 0.739974 \\
\hline
\end{tabular}

to consider additionally PCMs with many large errors as well as with many small and one large error. In our simulations we adopted these frameworks, however with some changes that made them more representative and realistic. First, the simulations described by Choo and 
Wedley (2004) and Lin (2007) were based only on one known priority vector. Moreover, the vector was not normalized and thus the observed average errors cannot be compared with errors corresponding to other vectors having different dimensions and priority values. In order to make the results more representative in our simulations we used the entire AHP framework with different number of criteria and different number of alternatives comprising random normalized priority vectors - in the most simple AHP framework considered, with three levels: goal, criteria and alternatives. There are four such priority vectors and we simulated 100 such frameworks perturbing every individual priority vector within each framework 100 times. Another difference is that we also took into account the rounding errors. Therefore the randomly disturbed ratios were rounded to the closest values from Saaty's scale. Such simulation framework seems to be more realistic and reliable, because in the AHP procedure decision makers are always expected to express their opinions on a given scale, and this operation is an additional and practically unavoidable source of error (Dong et al., 2008). In our simulations we studied the performance measures for chosen prioritization methods for four classes of probability distributions of the perturbation factors: uniform, lognormal, truncated normal and gamma. As we can notice from the entire simulation framework, we have grounds to claim that as long as reciprocity of PCMs is imposed (the requirement embedded in the AHP), it is not justified to claim that the REV is the dominant method among others as it was done by Saaty and $\mathrm{Hu}$ (1998). Additionally, exceptionally important seems to be the fact that the REV is as the rule worse in its performance measured by SRCC as long as reciprocity of PCMs is forced within the AHP in all researched scenarios. Our research clearly prove that the REV is not as effective as other arbitrarily chosen methods presented here in the process of unique capturing of the ratio scale rank order inherent in inconsistent pairwise comparison judgments as long as reciprocity of PCMs is forced in the AHP what stands in opposition to the theorem presented by Saaty and $\mathrm{Hu}$ (1998). To recapitulate, the REV surely is not the only valid method for deriving the priority vectors from a reciprocal pairwise comparison matrices, what was now verified from the perspective of order preservation concept within the entire AHP framework comprising inconsistent matrices. This seems very important from the viewpoint of multicriteria decision making in the field of scientific management because even if variability in ranks does not occur for individual judgment for different methods applied, it may still occur in the overall ranking of the final alternatives due to the multicriteria process itself. Because REV can give less credible rankings than other methods here presented, it is advised to use them instead of the REV, especially because they operate quite well also in the conditions where the REV stands down.

\section{Conclusions}

For a credible multicriteria decision making which support methodology, delivering unique answers, seems to be a crucial issue from the perspective of the entire concept validity. However, it is known that the analytic hierarchy process (AHP), as the prioritization and choice theory, can provide different priority vectors, depending on the method applied during the realization of the process. It is also known that from the very long time we are being convinced that only one approach in this matter is the only one and no other qualifies, that is principal right eigenvector method (REV). However, this approach finds itself to have serious drawbacks and some flaws. In this research we disclosed some fundamental issues within this field thanks to computer simulations for the whole AHP framework (as opposed to single matrix simulation research). The result verifies the statement that the right eigenvector method is not unique as long as reciprocal pairwise comparison matrices are concerned (what is embedded in the AHP process) and when it could be (nonreciprocal cases) it is impoverished by the lack of inconsistency measure in this case, which is an indispensable element of the entire AHP concept. Thus, especially in multicriteria decision making problems embedded in management processes we advise the application (together with the AHP) of other available methods like for example logarithmic utility approach to eigenvector method which is generally less complicated and more intuitive solution as well provides valid inconsistency measure for both reciprocal and nonreciprocal PCMs.

\section{REFERENCES}

Aguaron J, Moreno-Jimenez JM (2003). The Geometric consistency index: Approximate thresholds. Eur. J. Oper. Res. 147(1):137-145.

Bana e Costa CA, Vansnick J-C (2008). A critical analysis of the eigenvalue method used to derive priorities in AHP. Euro. J. Oper. Res. 187:1422-1428.

Basak I (1998). Comparison of statistical procedures in analytic hierarchy process using a ranking test. Math. Comp. Model. 28:105118.

Belton V, Gear AE (1983). On a shortcoming of Saaty's method of analytic hierarchies. Omega 11(3):228-230.

Bhushan N, Ria K (2004). Strategic Decision Making: Applying the Analytic Hierarchy Process. Springer-Verlag London Limited, London.

Bozóki S, Rapcsák T (2008). On Saaty's and Koczkodaj's inconsistencies of pairwise comparison matrices. J. Glob. Optim. 42:157-175.

Bryson N (1995). A goal programming method for generating priority vectors. J. Oper. Res. Soc. 46:641-648.

Budescu DV, Zwick R, Rapoport A (1986). Comparison of the analytic hierarchy process and the geometric mean procedure for ratio scaling. Appl. Psychol. Measure. 10:69-78.

Chamodrakas I, Batis D, Martakos D (2010). Supplier selection in electronic marketplaces using satisfying and fuzzy AHP. Expert Syst. Appl. 37:490-498.

Chen MK, Wang S-C (2010). The critical factors of success for information service industry in developing international market: Using analytic hierarchy process (AHP) approach. Expert Syst. Appl. 37:694-704. 
Choo EU, Wedley WC (2004). A common framework for deriving preference values from pairwise comparison matrices. Comp. Oper. Res. 31:893-908.

Cook WD, Kress M (1988). Deriving weights from pairwise comparison ratio matrices: An axiomatic approach. Eur. J. Oper. Res. 37:355362.

Crawford G, Williams CA (1985). A note on the analysis of subjective judgment matrices. J. Math. Psychol. 29:387-405.

Dong Y, Xu Y, Li H, Dai M (2008). A comparative study of the numerical scales and the prioritization methods in AHP. Eur. J. Oper. Res. 186:229-242.

Farkas A (2011). Multi-Criteria Comparison of Bridge Designs. Acta Polytech. Hung. 1(8):173-191.

Grzybowski AZ (2010). Goal programming approach for deriving priority vectors - some new ideas. Sci. Res. Inst. Math. Comp. Sci. 1(9):1727.

Grzybowski AZ (2012). Note on a new optimization based approach for estimating priority weights and related consistency index. Expert Syst. Appl. 39:11699-11708.

Ho W (2008). Integrated analytic hierarchy process and its applications - A literature review. Eur. J. Oper. Res. 186:211-228.

Hovanov NV, Kolari JW, Sokolov MV (2008). Deriving weights from general pairwise comparison matrices. Math. Soc. Sci. 55:205-220.

Hummel M (2001). Supporting Medical Technology Development with the Analytic Hierarchy Process. The Netherlands: Rijksuniversiteit Groningen, Groningen.

Isaai MT, Kanani A, Tootoonchi M, Afzali HR (2011). Intelligent timetable evaluation using fuzzy AHP. Expert Syst. Appl. 38:37183723.

Ishizaka A, Labib A (2011). Review of the main developments in the analytic hierarchy process. Expert Syst. Appl. 11(38):14336-14345.

Kahraman C, Kaya I (2010). A fuzzy multicriteria methodology for selection among energy alternatives. Expert Syst. Appl. 9(37):62706281.

Kilincci O, Onal SA (2011). Fuzzy AHP approach for supplier selection in a washing machine company. Expert Syst. Appl. 38:9656-9664.

Lam KF, Choo EU (1995). Goal programming in preference decomposition. J. Oper. Res. Soc. 46:205-213.

Li S, Li JZ (2009). Hybridizing human judgment, AHP, simulation and a fuzzy expert system for strategy formulation under uncertainty. Expert Syst. Appl. 36:5557-5564.

Lin C-C (2006). An enhanced goal programming method for generating priority vectors. J. Oper. Res. Soc. 57:1491-1496.

Lin $C-C$ (2007). A revised framework for deriving preference values from pairwise comparison matrices. Eur. J. Oper. Res. 176:11451150.

Lipovetsky S, Conklin MM (2002). Robust estimation of priorities in the AHP. Eur. J. Oper. Res. 137:110-122.

Lipovetsky S, Tishler A (1997). Interval estimation of priorities in the AHP. Eur. J. Oper. Res. 114:153-164.

Merkin BG (1979). Group Choice. John Wiley \& Sons, New York.
Moy JW, Lam KF, Choo EU (1997). Deriving the partial values in MCDM by goal programming. Ann. Oper. Res. 74:277-288.

Rad A, Naderi B, Soltani M (2011). Clustering and ranking university majors using data mining and AHP algorithms: A case study in Iran. Expert Syst. Appl. 38:755-763.

Saaty TL (1980). The Analytic Hierarchy Process. McGraw-Hill, New York.

Saaty TL (1986). Axiomatic foundation of the analytic hierarchy process. Management Sci. 32:841-855.

Saaty TL (1990). How to make a decision: the analytic hierarchy process. Eur. J. Oper. Res. 48:9-26.

Saaty TL (2000). The Brain: Unraveling the Mystery of How it Works. RWS Publications, Pittsburgh, PA

Saaty TL (2001). Decision Making for Leaders. The Analytical Hierarchy Process for Decisions in a Complex World. RWS Publications, Pittsburgh.

Saaty TL (2003). Decision making with the AHP: Why is the principal eigenvector necessary. Euro. J. Oper. Res. 145:85-91.

Saaty TL (2006). Fundamentals of Decision Making and Priority Theory with the Analytic Hierarchy Process. RWS Publication, Pittsburgh, PA.

Saaty TL, Hu G (1998). Ranking by Eigenvector versus other methods in the Analytic Hierarchy Process. Appl. Math. Lett. 11(4):121-125.

Saaty TL, Vargas LG (1984). Comparison of eigenvalue, logarithmic least square and least square methods in estimating ratio. J. Math. Model. 5:309-324.

Saaty TL, Vargas LG (2000). Models, Methods, Concepts and Applications of the Analytic Hierarchy Process. Kluwer Academic Publishers, Boston.

Saaty TL, Vargas LG (2006). Decision Making with the Analytic Network Process: Economic, Political, Social and Technological Applications with Benefits, Opportunities, Cost and Risks. Springer, New York.

Schmold D, Kangas J, Mendoza G, Pesonen M (2001). The Analytic Hierarchy Process in Natural Resource and Environmental Decision Making. Kluwer Academic Publishers, Dordrecht, Boston, London.

Sun L, Greenberg BS (2006). Multiple group decision making: optimal priority synthesis from pairwise comparisons. J. Optm. Theory Appl. 130(2):317-338

Vaidya OS, Kumar S (2006). Analytic hierarchy process: An overview of applications. Euro. J. Oper. Res. 169:1-29.

Vidal L-A, Marle F, Bocquet J-C (2011). Using a Delphi process and the Analytic Hierarchy Process (AHP) to evaluate the complexity of projects. Expert Syst. Appl. 38:5388-5405.

Xu ZS (2004). Goal programming models for obtaining the priority vector of incomplete fuzzy preference relation. Inter. J. Approx. Reason. 36:261-270.

Zahedi F (1986). A simulation study of estimation methods in the analytic hierarchy process. Socio-Econ. Plann. Sci. 20:347-354. 